\title{
L’AFFICHAGE À MONCTON : MIROIR OU MASQUE ?
}

\author{
Annette Boudreau et Lise Dubois
}

Université de Moncton

\section{Résumé}

L'étude sur laquelle cet article se fonde combine trois types de démarches: d'abord, l'étude du paysage langagier par le biais de l'affichage public; des entretiens semidirectifs faits avec des Monctoniens francophones et anglophones ; des séances d'observation selon lesquelles les participants à l'étude ont été accompagnés dans leurs déplacements quotidiens. Les questionnements au centre de la recherche sont les suivants: le citoyen navigue-t-il différemment dans ce paysage selon qu'il est francophone ou anglophone? Quels sont les indices visibles et audibles qui lui permettent de faire le choix de langue qui lui profitera le plus selon la situation dans laquelle il se trouve ? Est-ce que l'affichage public participe de la négociation de langues que font quotidiennement les Monctoniens? Quels sont les discours qui circulent autour de la question de l'affichage à Moncton et contribuent-ils à maintenir les pratiques existantes ou, au contraire, à les changer ?

\section{Abstract}

The research project on which this paper is based combines a three-pronged approach to the study of the city: the linguistic landscape was surveyed through public signage, interviews were conducted with Francophone and Anglophone Monctonians and some of the participants were accompanied throughout their daily activities by a research assistant. The research project attempts to answer the following questions: does a francophone resident of Moncton circulate through the city differently than an anglophone resident? What are the visible and audible clues that allow 
Monctonians to chose the language that will benefit them the most according to the communication situation in which they find themselves? Is the public signage part of the ongoing daily negociations on what language to use in Moncton? What are the various discourses on the question of bilingual signage and do they contribute to linguistic maintenance or change?

\begin{abstract}
notre ville au drôle de nom cette ville au nom de notre bourreau le général monckton qui brûle éternellement en enfer aujourd'hui nous composons une cartographie de nos besoins à l'appel du dedans comme directive devant notre perte et notre entêtement les modes s'épuisent à nous solliciter nous sommes déjà ailleurs
\end{abstract} Gérald Leblanc (1995 : 118)

\section{Introduction}

Décrire comment se vivent les contacts des langues et des locuteurs de ces langues dans des espaces urbains n'est pas chose simple puisque la ville est le lieu par excellence de l'hétérogénéité et de dynamiques linguistiques souvent contradictoires où les locuteurs de langues différentes ont à se trouver ou à se fabriquer des espaces où dire (ou vivre) leur identité linguistique. Si cela va de soi pour les locuteurs de langues dominantes, il en est parfois tout autre pour les locuteurs de langues dominées. En effet, les espaces où se dire dans sa langue pour ces derniers sont souvent des espaces qui ne sont pas donnés d'emblée : ils sont souvent créés par les locuteurs eux-mêmes et c'est dans ces espaces inventés, dans ces interstices, que les locuteurs minorisés trouvent un lieu où s’exprimer.

Ces lieux de la parole dominée ne sont pas toujours identifiables dans le paysage linguistique urbain. En effet, pour les étrangers qui arrivent dans une ville, l'affichage peut fournir des indices de la répartition linguistique de la population, mais il est rarement le miroir de la situation 
linguistique réelle. Il est plutôt le miroir des inégalités sociales et, souvent, le masque sous lequel se cachent des luttes de pouvoir autour d'enjeux matériels et symboliques de toutes sortes, y compris l'occupation spatiale de la ville. De plus, l'affichage est révélateur des représentations que les locuteurs entretiennent à l'égard des langues qui circulent dans la ville, représentations liées aux idéologies linguistiques qui façonnent les discours sur les langues.

La question de la langue de l'affichage public dans un pays officiellement bilingue comme le Canada n'est certes pas nouvelle ; elle a fait l'objet d'un grand nombre d'études, de rapports et de relevés, surtout dans le cadre de l'application de la Charte de la langue française au Québec, laquelle a agi dès sa mise en vigueur dans le domaine de l'affichage. Au Nouveau-Brunswick, les lois sur les langues officielles fédérale et provinciale ${ }^{1}$ obligent les ministères et organismes gouvernementaux d'afficher dans les deux langues. En revanche, la Société des Acadiennes et Acadiens du Nouveau-Brunswick (SAANB ${ }^{2}$ ) mène depuis de nombreuses années diverses campagnes qui visent à inciter les commerçants des régions bilingues de la province à afficher davantage dans les deux langues. Cette campagne connaît un succès particulier à Dieppe, ville qui fait partie du Grand Moncton, où l'affichage bilingue augmente d'année en année grâce aux efforts concertés de la SAANB et de l'administration municipale ${ }^{3}$. Dans le cadre des analyses et des débats qui motivent ces campagnes, c'est surtout la fonction symbolique de l'affichage bilingue qui a été mise de l'avant comme composante indispensable de la vitalité perçue et vécue de la langue minoritaire et comme fenêtre sur le changement linguistique visé, et ce, dans une perspective éminemment aménagiste (Landry et Bourhis, 1997; Bourhis et Landry, 2002).

La sociolinguistique urbaine aborde la question de l'affichage sous un autre angle. En effet, dans le cadre de cette discipline qui se préoccupe surtout d'étudier «la mise en mots de la co-variance entre la structure spatiale signifiante et la stratification sociolinguistique » (Bulot, 2002: 94), l'affichage constitue l'un des multiples discours épilinguistiques en circulation qui visent à "marquer l'occupation et l'appropriation de l'espace urbain par des groupes sociaux » (Ibid.). Elle vise également à cerner, d'une part, les conditions historiques, sociales et économiques 
dans lesquelles ces discours sont produits et, d'autre part, les effets conjugués de l'espace sur les comportements et pratiques linguistiques et, corollairement, des discours sur l'espace (Ibid.; Bulot et Veschambre, 2004). Autrement dit, la sociolinguistique urbaine appréhende les affiches comme des discours autour desquels s'articulent des luttes de pouvoir entre groupes en ce qui a trait à l'occupation spatiale de la ville et se définit l'appartenance de l'espace. Dans une ville bilingue où il y a non seulement contact des langues en présence, mais aussi concurrence, réelle ou perçue entre elles (voir infra; Dubois, 2003), les affiches sont des symboles de l'appropriation des espaces et, dans certains cas, les emblèmes de cette appropriation.

Dans la ville officiellement bilingue de Moncton au NouveauBrunswick, il est parfois difficile pour l'étranger qui y arrive de « voir » le statut bilingue de la ville ${ }^{4}$. Le paysage linguistique est en effet dominé par l'anglais ${ }^{5}$ : dans cet article, nous examinerons comment s'opère cette domination par l'anglais dans l'affichage, ainsi que les modalités selon lesquelles le français s'insère dans le paysage linguistique. Nous tenterons également de faire ressortir les liens entre le paysage linguistique de Moncton et les représentations linguistiques d'anglophones et de francophones qui circulent quotidiennement dans cet environnement. Ce texte rendra également compte des différents types de bilinguisme qui se superposent dans la ville de Moncton, reflet des diverses juridictions qui y sont présentes (fédérale, provinciale et municipale) et des pratiques distinctes exercées sous chacune d'elles. Les questionnements au centre de la recherche sont les suivants : le citoyen s’oriente-t-il différemment dans le paysage urbain monctonien selon qu'il est francophone ou anglophone? Quels sont les indices visibles (et audibles) qui lui permettent de faire le choix de langue qui lui profitera le plus selon la situation dans laquelle il se trouve ? Est-ce que l'affichage public participe de la négociation de langues que font, parfois plusieurs fois par jour, les Monctoniens, surtout les francophones? Quels sont les discours qui circulent autour de la question de l'affichage à Moncton et contribuent-ils à maintenir les pratiques existantes ou, au contraire, à les changer ? Existe-t-il des espaces-nous et des espaces-ils ${ }^{6}$ à Moncton et comment sont-ils repérés par les locuteurs ? 


\section{Cadre et méthodologie}

Cette recherche s'inscrit dans un projet plus large sur la construction discursive de l'espace francophone minoritaire au Canada ${ }^{7}$ qui avait comme objectif d'étudier comment les acteurs sociaux francophones de deux régions canadiennes, l'Ontario et l'Acadie, expriment leur francité, se définissent comme francophones et circonscrivent l'espace dans lequel ils se disent francophones. La méthodologie du projet s'est déployée sur trois types de démarches: l'analyse contextuelle et documentaire, l'analyse du discours au moyen d'entretiens semi-directifs et l'observation des interactions dans les sites de recherche (voir Heller et Labrie, 2003). Cette étude a démontré que la «francité » dans les communautés minoritaires canadiennes était caractérisée par son éclatement, sa fluidité et sa complexité (Ibid., : 405). Si la francophonie canadienne s'est organisée au fil des années autour d'espaces sociaux, plus particulièrement depuis un peu plus d'une quarantaine d'années, ces derniers sont toujours sujets à des "contraintes matérielles et sociales » (Ibid., : 407) qui font en sorte que la pleine réalisation de la francité en milieu minoritaire est impossible (Ibid.). De là, la redéfinition des discours identitaires et la négociation constante entre minorités, groupes dominants et État. Si cette recherche nous a permis d'appréhender les discours des francophones sur la francité, elle nous a également amenées à nous interroger sur les discours que le groupe dominant (les anglophones) entretient quant à la même question, soit la francité.

Il sera donc question dans cet article de comparer les représentations linguistiques d'acteurs sociaux francophones et anglophones à la lumière des pratiques entourant l'affichage public de Moncton et relativement à la construction d'espaces identitaires dans la ville. Dans le cadre du sousprojet sur les représentations des anglophones à l'égard du français et des francophones, l'équipe de recherche a effectué des entrevues auprès de 18 anglophones ${ }^{8}$ entre 2001 et 2004 selon une grille semblable à celle suivie pour le projet initial (Prise de parole) qui comportait trois parties principales: la trajectoire personnelle du participant, sa vie professionnelle et/ou engagement social actuels et sa perception de l'avenir du Nouveau-Brunswick, en lien avec sa conscience de l'existence des francophones et du français. Ces participants ont été choisis suivant la 
méthode éprouvée dans le cadre du projet initial : ils occupent des postes de direction dans différents secteurs, notamment les médias, le système scolaire, le monde des affaires, le monde associatif, le secteur municipal et les soins de santé. Ces acteurs sociaux sont ou bien nés à Moncton et y habitent toujours, ou bien nés ailleurs mais y vivent depuis le début de leur vie professionnelle. Pour ce qui est de l'analyse comparative, nous nous fondons d'une part sur le corpus d'entretiens constitué lors du projet Prise de parole, dans le cadre duquel nous avons fait 46 entretiens $^{9}$ et, d'autre part, sur la participation de deux francophones qui ont accepté d'être interviewés et accompagnés dans le cadre de la troisième phase du projet L'image du français chez les anglophones des Maritimes.

Au terme de la première phase de ce dernier projet, nous avons été amenées à conclure qu'il existe effectivement des différences entre les représentations que se font les francophones à l'égard du bilinguisme et des questions qui l'entourent et celles des anglophones, faisant de Moncton un lieu de luttes discursives surtout en ce qui a trait à l'accès à la compétence bilingue, laquelle procure des ressources matérielles et symboliques (voir Boudreau \& Dubois, 2003 ; Dubois, 2003). Depuis 2004, nous avons ajouté un volet d'observation à l'entretien selon lequel le participant est observé dans ses déplacements quotidiens sur une période de 24 heures dans le but de découvrir si le découpage linguistique de l'espace urbain joue dans les déplacements quotidiens du citoyen moyen ; en d'autres termes, les différences sur le plan représentationnel structurent-elles la vie des citoyennes et citoyens de Moncton et s'actualisent-elles dans leur quotidien?

Dans cet article, nous présentons un bref examen du paysage linguistique par le biais de l'affichage public. Nous avons fait un relevé systématique de l'affichage public le long de deux axes spatiaux importants du centre-ville de Moncton: le premier, l'axe St.George/Archibald-avenue de l'Université dans lequel on compte une forte concentration d'institutions acadiennes et francophones et le second, la rue «principale », qui porte le nom officiel de rue Main Street. Ensuite, nous repérons les discours sur l'affichage public et, plus particulièrement, nous analysons comment est créée la ville dans le discours de Monctoniens francophones et anglophones à partir des corpus d'entretiens semi-directifs que nous recueillons depuis 1996 auprès de francophones et 
depuis 2001 auprès d'anglophones. Tel que mentionné ci-dessus, certains de ces entretiens ont été enrichis de séances d'observation pendant lesquelles les participants ont été accompagnés dans leurs déplacements quotidiens durant une journée. Nous présentons ici les premiers résultats d'une enquête dont les objectifs sont directement liés à l'espace, tout en s'inscrivant dans le droit fil des objectifs généraux et comparatifs de la recherche plus large. Finalement, nous verrons comment le contact des langues à Moncton est représenté dans quelques textes d'écrivains.

Toutefois, avant de présenter les résultats du relevé de l'affichage, il importe de faire un survol rapide de ce qui est communément appelé l'espace francophone de Moncton.

\section{L'espace francophone de Moncton}

Au cours des cinquante dernières années, grâce à des facteurs que nous évoquerons brièvement ci-dessous, l'espace francophone à Moncton s'est considérablement étendu et vitalisé. Cet espace francophone à Moncton n'est pas associé à un territoire nettement circonscrit, c'est-à-dire à un quartier précis de la ville. Il est plutôt, à l'image de la francophonie canadienne dans son ensemble, tel que nous l'avons mentionné ci-dessus, fluide, éclaté : il se réalise en grande partie là où les francophones exercent un certain pouvoir et peuvent exprimer leur francité. C'est le cas, par exemple, des institutions acadiennes créées au fil des années et dans lesquelles est ancrée l'expression identitaire : l'église, certains médias, le monde associatif et, plus récemment, l'école, l'université et le secteur culturel et artistique. Comme l'a avancé Anne Gilbert, l'espace francophone est «le résultat de la juxtaposition des réseaux sociaux devant relier entre eux les lieux de la francophonie sur une certaine durée » (1999 : 16). Toujours selon Gilbert, l'espace francophone est aussi le résultat de l'interaction et de l'échange entre ces lieux «qui lui permettent de transcender la distance et la dispersion » (Ibid. : 17).

Rappelons brièvement les secteurs où les francophones de Moncton ont fait d'importants acquis qui viennent consolider et dynamiser l'espace francophone monctonien (pour des analyses plus détaillées, voir Boudreau et Dubois, 2002 ; Dubois, 2003). D'abord, l'histoire des revendications des Acadiens tout au long du $\mathrm{XX}^{\mathrm{e}}$ siècle se caractérise par la volonté de créer des espaces sociaux francophones en vue de favoriser la survie de la 
langue et de la culture acadiennes, ce qui n'empêche pas que certaines institutions acadiennes offrent aujourd'hui des services dans les deux langues, tout en maintenant le français comme langue de travail ou de communication interne. Les institutions qui découlent de ce mouvement sont fort bien connues et font partie intégrante de la grande complétude institutionnelle dont jouit la société acadienne. Nous n'en retenons que quelques-unes aux fins de ce texte. On ne peut faire abstraction des répercussions considérables de la fondation de l'Université de Moncton, université entièrement de langue française, sur plus d'un plan : économique ${ }^{10}$, ulturel $^{11}$ et social. Sur le plan des affaires, les Acadiens font preuve d'un fort esprit entrepreneurial et lancent des entreprises lucratives dans une grande gamme de secteurs (Allain, à paraître). Les gens d'affaires acadiens et francophones se rassemblent au sein du Conseil économique du Nouveau-Brunswick, créé en 1979, lequel représente un autre réseau qui contribue à la vitalité de l'espace francophone. Enfin, sur le plan des médias, les Acadiens ont accès à la gamme complète des médias dans leur langue: radio et télévision publiques, communautaires et privées et un quotidien.

À Moncton, plus que nulle part ailleurs au Canada, les régimes linguistiques se superposent: les bilinguismes officiels des administrations fédérale, provinciale et municipale s'actualisent dans l'affichage public. En effet, on y trouve, comme dans toutes les régions et villes du Canada, l'affichage bilingue des bureaux fédéraux régi par la Loi sur les langues officielles du Canada. De plus, l'affichage dans les bureaux provinciaux est régi par la Loi sur les langues officielles $d u$ Nouveau-Brunswick, à titre de seule province officiellement bilingue au Canada. Par conséquent, tous les services au citoyen qui relèvent des compétences fédérale et provinciale sont obligatoirement annoncés dans les deux langues. Seule exception à cette règle, le monde scolaire, qui, au Nouveau-Brunswick, est structuré selon le principe de la dualité linguistique. Résultat : les écoles et les collèges de la province constituent des espaces linguistiques homogènes ou bien français, ou bien anglais. Enfin, puisque Moncton est la première ville à s'être reconnue officiellement bilingue au Canada (2002), l'administration municipale doit veiller à ce que ses services soient également annoncés dans les deux langues. Cette superposition de régimes linguistiques est un autre facteur qui a contribué, au cours des cinquante dernières années, non seulement à 
la croissance et à la vitalisation de l'espace francophone à Moncton, mais aussi à la visibilité, bien que relative, de la présence d'une communauté francophone.

Quelques mots sur les marchés linguistiques officiels du NouveauBrunswick: ces derniers sont occupés par deux langues légitimes, le français et l'anglais, cette dernière prenant toujours, dans l'ensemble, une plus grande place (dans l'appareil gouvernemental, le secteur municipal, les échanges commerciaux, etc.), malgré le fait que les deux langues soient langues co-officielles ${ }^{12}$. De même, malgré ce statut officiel sur le plan provincial, le bilinguisme qui s’y pratique reste asymétrique. Si $70 \%$ des francophones parlent anglais, seulement $15 \%$ des anglophones parlent français assez couramment pour soutenir une conversation (Statistique Canada, 2001). Fait important à mentionner : la forte majorité des francophones de Moncton (du Grand Moncton, pourrait-on préciser) parlent anglais sans accent et sans intonation révélateurs du fait qu'ils soient francophones, d'où l'incertitude fréquente dans l'interaction entre étrangers quant à la langue à utiliser.

\section{Espace francophone et affichage public : une rupture}

Si l'espace francophone monctonien est reconnu comme étant un espace vital et dynamique, il est parfois difficile de le constater à «l'œil nu ». Tel que mentionné ci-dessus, l'affichage public qui témoignerait de cette vitalité, voire de son existence, est encore aujourd'hui largement dominé par l'anglais. Le relevé systématique de l'affichage public le long de deux axes spatiaux importants de Moncton pourrait laisser croire à une communauté francophone peu présente dans la vie quotidienne de la ville, c'est-à-dire dans l'espace vécu. Le tableau 1 présente le recensement des affiches repérées le long des axes commerciaux décrits ci-dessus selon la typologie suivante :

1. les affiches unilingues anglaises où n'apparaît aucune unité lexicale en français ;

2. les affiches partiellement bilingues, anglo-dominantes, où la raison sociale est en anglais, et le descriptif du commerce est dans les deux langues ; 
Tableau 1 : Typologie de l’affichage public à Moncton (Printemps 2005) 
3. le contraire de la catégorie précédente, les affiches partiellement bilingues, franco-dominantes, où la raison sociale est en français, et le descriptif du commerce dans les deux langues ;

4. les affiches obligatoirement bilingues en raison d'une loi quelconque, fédérale, provinciale ou municipale ;

5. les affiches entièrement bilingues, sans obligation juridique quelconque. Certaines raisons sociales qui se trouvent dans cette catégorie n'appartiennent ostensiblement à ni l'une ni l'autre langue, que ce soit sur le plan du lexique ou de la structure ;

6. les affiches unilingues françaises.

\section{L'axe St.-George-Archibald/avenue de l'Université ${ }^{13}$}

Le premier axe recensé se situe dans le quartier où l'on retrouvait avant les années soixante le plus grand nombre de familles francophones dans la ville de Moncton (Durand, 2004), c'est-à-dire l'axe St.-GeorgeArchibald/avenue de l'Université. Aujourd'hui, si les familles francophones y sont moins nombreuses, on compte le long de cet axe une concentration relativement importante d'institutions acadiennes et francophones (notamment la cathédrale acadienne, le siège social de Radio-Canada, le centre hospitalier francophone et l'université qui est unilingue française).

Nous y avons relevé 112 affiches, dont $53 \%$ sont unilingues anglaises. La très forte majorité de ces affiches unilingues anglaises représentent une grande gamme de petites entreprises d'envergure locale, c'est-à-dire des petits commerçants qui exploitent un seul site, tel un salon de coiffure, une école d'arts martiaux, un magasin d'articles spécialisés (entre autres lunettes, appareils photo, antiquités), un dépanneur, un restaurant, etc., ou encore des professionnels qui n'ont qu'un seul bureau, tels un dentiste, un comptable/conseiller financier, un avocat, un assureur, etc. Sur les 59 affiches repérées dans cette catégorie, on ne compte que 5 entreprises qu'on pourrait qualifier de "moyennes » ou de "grandes », c'est-à-dire des succursales d'entreprises à rayonnement régional, national ou international, telles des coopératives d'épargne et de crédit ayant des succursales dans la province ou dans la région de l'Atlantique et une 
entreprise de location de voitures ayant des succursales partout au monde, etc.

Dans la deuxième catégorie, soit les affiches partiellement bilingues, anglo-dominantes, 11 des 13 affiches repérées annoncent des entreprises locales et seulement 2 sont des entreprises d'envergure nationale. La caractéristique saillante de cette catégorie est que la raison sociale est en anglais, avec les marques morphosyntaxiques propres à cette langue (par ex., le possessif anglais qui se marque au moyen de l'apostrophe $+\mathrm{s}$ : 's), mais le commerçant ou l'entreprise a choisi d'annoncer ses produits ou ses services dans les deux langues. L’inverse n’a pas été repéré : aucune raison sociale en français avec le descriptif dans les deux langues.

Un certain nombre d'affiches sont entièrement bilingues (12): les lieux fédéraux (3) ; les lieux provinciaux (6) ; des organismes sans but lucratif d'envergure nationale (2) et une société d'État. Comme on le sait déjà, ces organismes ont une obligation juridique d'afficher dans les deux langues et, selon les pratiques d'affichage bilingue mises en œuvre au Canada et au Nouveau-Brunswick depuis l'adoption des lois sur les langues officielles, toutes les unités lexicales composant l'affiche sont présentes dans les deux langues ou bien en superposition, ou bien en présentation côte à côte. On trouve également dans cette catégorie un certain nombre d'entreprises (17), dont 12 d'envergure locale et 5 d'envergure nationale ou régionale, dont l'affichage est entièrement bilingue. Sur le plan linguistique, il est intéressant de noter les innovations lexicales qu'on peut faire en milieu bilingue. Par exemple, dans l'appellation Christian Resources Centre Ressources chrétiennes, on privilégie la graphie britannique de centre plutôt que la graphie plus commune en anglais nord-américain de center pour qu'elle puisse servir de charnière entre l'anglais et le français. Dans un autre exemple, on sacrifie les accents français pour permettre ce même type de construction avec charnière bilingue: École de musique Metro Moncton School of Music (l'accent aigu est omis sur Metro). D'autres constructions à charnière: Imprimerie Maritime Press (le spécifique Maritime ne changeant pas de graphie de l'anglais au français), ou encore Maison du Commerce House. Enfin, fait digne de mention : une des banques à charte canadienne affiche une devanture bilingue dans la rue St.-George, mais unilingue anglaise dans la rue Main. 
La dernière catégorie, les affiches unilingues françaises, à part une exception ${ }^{14}$, sont toutes des marqueurs d'institutions acadiennes ou francophones, lesquelles témoignent de l'existence d'espaces sociaux francophones ancrés, pour la plupart, dans cette partie de la ville depuis un certain nombre d'années et constituent des établissements clés dans la structuration de la vie francophone à Moncton : l'église, l'université, une coopérative artistique, une coopérative d'épargne et de crédit d'envergure régionale, un organisme réunissant les gens d'affaires acadiens et francophones. Ces institutions acadiennes constituent le terreau de l'identité acadienne.

Qu'est-ce que révèle ce relevé des affiches sur les devantures le long de cet axe commercial de Moncton ? D'abord, bien qu'un certain nombre de petits commerçants locaux, environ une vingtaine, s'affichent entièrement ou partiellement dans les deux langues, la majorité, soit 55, qu'ils soient francophones ou anglophones, sont dans une large mesure assez indifférents à l'affichage bilingue. Faut-il croire qu'ils ne perçoivent aucun avantage commercial à l'affichage bilingue ? En fait, ce sont les intérêts locaux qui ont le moins recours au français dans leur devanture, puisque les entreprises d'envergure nationale, sans doute en raison de leurs pratiques d'affichage au Québec, sont plus enclines à afficher dans les deux langues (7 entreprises d'envergure nationale sur 11 utilisent le français dans leur affichage). Puis, l'affichage unilingue français le long de ces deux rues est révélateur de la présence historique de la communauté française dans cette partie de la ville, mais depuis dispersée sur tout le territoire urbain. Si l'affichage unilingue français le long de cet axe ne témoigne pas nécessairement d'une vie communautaire francophone ancrée dans le territoire, il semble bien y avoir une reconnaissance tacite de la présence francophone dans cette partie de la ville, qu'elle soit plutôt institutionnelle que communautaire. Le fait que les panneaux de signalisation le long de la rue de l'Université sont francodominants (ARRÊT étant imprimé au-dessus de STOP dans la rue de l'Université), ce qui est contraire à la pratique ailleurs dans la ville, constitue à notre avis la reconnaissance officielle de la présence d'institutions acadiennes ou francophones dans cette partie de la ville. 


\section{L'axe rue Main ${ }^{15}$}

Le second axe qui a fait l'objet de ce relevé révèle l'existence d'une dynamique assez différente de celle décrite ci-dessus. En effet, on retrouve le long de cette rue une vie urbaine plus marquée, grâce aux nombreux restaurants, y compris cafés et terrasses, et aux nombreux grands bureaux qui y sont installés. Il est facile d'associer l'ambiance qui se dégage de cette rue un jour de semaine à l'essor économique que connaît Moncton depuis une dizaine d'années. Bien que la proportion d'affiches unilingues anglaises (52\%) et le profil de ces entreprises ${ }^{16}$ soient comparables le long des deux axes, la proportion de commerçants et de professionnels affichant entièrement dans les deux langues est deux fois plus importante dans la rue Main (15\% dans l'axe St.George/Archibald-avenue de l’Université contre $30 \%$ dans la rue Main). Plusieurs raisons peuvent expliquer cette différence. D’abord, dans la rue Main, les cafés et terrasses qui attirent une clientèle régulière surtout le midi sont plus nombreux à afficher une raison sociale à appartenance linguistique ambiguë (p. ex. Joe Moka ; Pastalli ; Capitol ; Cafér Cognito). Ces affiches ont été classées dans la catégorie d'affiches entièrement bilingues puisqu'elles peuvent se lire aussi bien en français qu'en anglais. De plus, une lecture des noms sur les affiches annonçant des bureaux où l'on pratique une profession libérale révèle qu'il y a plus de francophones professionnels qui exercent dans la rue Main que dans la rue St.-George. Parmi les affiches entièrement bilingues (31 au total), on trouve également les organismes gouvernementaux fédéraux et provinciaux (5) dont l’affichage est régi par la loi.

Sur les 71 affiches relevées, aucune affiche unilingue française n’a été repérée. À part une société d'assurance et de placement généralement associée à la société acadienne, qui affiche dans les deux langues contrairement à la pratique dans l'autre axe dont il est question, on ne retrouve pas sur cet axe la même concentration d'institutions acadiennes que sur l'axe St.-George/Archibald-avenue de l'Université.

Il nous apparaît assez clairement qu'il y a effectivement une rupture entre la vitalité et l'importance économique et sociale de l'espace francophone de Moncton et les pratiques d'affichage. Ce relevé révèle le degré de ténacité des pratiques d’affichage locales devant un changement social sur lequel il est difficile de fermer les yeux : le statut officiellement 
bilingue de la ville, ville longtemps reconnue pour son hostilité au fait français ; le revirement économique largement attribué dans le discours officiel à la communauté acadienne ; la vitalité culturelle et artistique, également reconnue comme fait surtout francophone, sont tous des éléments indicateurs d'un changement social profond en opération, lequel reste largement invisible dans l'affichage commercial. Nous tenterons d'approfondir ci-dessous cette réticence en analysant les discours et les représentations et, partant, les idéologies, que nous avons repérés dans les entretiens de personnes qui façonnent le tissu urbain monctonien.

En revanche, tel qu'indiqué précédemment, les institutions acadiennes tiennent à s'afficher en français, témoignant ainsi d'une vie francophone institutionnelle bien ancrée à Moncton et explicitement reconnue par les autorités municipales. Cependant, le relevé ne montre pas qu'il existe une forte vie communautaire francophone qui soit géographiquement ancrée autour d'elles. Sans doute peut-on en partie expliquer ce phénomène par la configuration territoriale même des villes nord-américaines.

Depuis les années soixante-dix, la plupart des villes nord-américaines, hormis les très grandes villes, se caractérisent par le décentrement (Baudrillard, 1986 ; Vincent, 1992); elles sont étendues sur des kilomètres, dont les centres sont désertés au profit des centres commerciaux souvent construits en banlieue ou en périphérie. En effet, contrairement à la plupart des villes présentées dans ce numéro, les villes nord-américaines moyennes ne sont pas toutes dotées d'un centre-ville ou de quartiers clairement identifiés, différents les uns des autres. Selon Vincent, géographe,

Le vent de prospérité qui souffle (depuis les années cinquante) sur l'Amérique du Nord amène à la population des salaires appréciables et des heures de travail réduites [...] ; [et que] cette prospérité se traduit également par une mobilité plus grande, elle-même associée à la diffusion plus large de l'automobile, et par la possibilité de devenir propriétaire d'une unité de logement, plutôt que locataire (Vincent, 1992 : 3). 
Dotés de voitures, les gens s'installent en banlieue afin de profiter des plus grands espaces et des logements plus économiques qui leur sont offerts. La ville nord-américaine ne peut se concevoir qu'en termes d'espaces éclatés avec des longues rues très étendues davantage conçues pour les autos que pour les piétons. La vie citadine dans ces espaces n’est donc pas organisée autour d'un centre constituant le cœur de la ville et le lieu de rencontres. Moncton n’échappe pas à ce phénomène : la ville est dotée d'un petit centre-ville certes mais les centres commerciaux situés aux extrémités de la ville sont les lieux de rassemblement des citoyens.

Cependant, au-delà de ces espaces matériels et tangibles qui ne représentent qu'un aspect de la réalité urbaine qui se donne à voir, on peut affirmer, avec Mondada, que les villes existent surtout dans et par les discours des gens qui l'habitent et prennent forme à travers ces discours qui les constituent :

L’urbanité est moins définie en termes de
propriétés matérielles (p. ex. en termes de
population, de transports, de flux, d'échanges),
qu'en termes de propriétés symboliques : le
caractère urbain de la ville, ce qui la constitue dans
son urbanité, n'est pas réductible à sa morphologie
ou à sa concentration d'activités économiques,
mais dépend des discours qui portent sur elle et
qui, en circulant dans des réseaux de plus en plus
étendus, en se solidifiant dans des versions de plus
en plus évidentes, se sédimentent dans les
représentations et dans la matérialité même
(Mondada, $2000: 29-30$ ).

Ce sont justement les discours qui portent sur Moncton que nous abordons dans la partie 4 qui suit.

\section{L'affichage public: représentations contradictoires}

Pour appréhender la valeur symbolique et matérielle qu'accordent les Monctoniens francophones à l'affichage bilingue, il faut d'abord saisir non seulement la valeur qu'ils accordent au bilinguisme dans son ensemble, mais aussi les conditions sociales et politiques dans lesquelles ce bilinguisme a été institué. Dans le discours des organismes et des 
acteurs sociaux acadiens des années 1960, les revendications pour le bilinguisme officiel étaient intimement associées aux revendications pour l'égalité de la société acadienne, c'est-à-dire sa participation égale à la vie sociale, politique et économique de la province (Heller et Labrie, 2003 : 9-39). Le bilinguisme officiel, avec ses traces visibles, constitue donc une reconnaissance explicite de l'existence même d'une communauté francophone au Nouveau-Brunswick (1969), et à Moncton (2002). Malgré les acquis considérables, l'affichage commercial dans les deux langues à Moncton reste, aujourd'hui, un enjeu politique de taille, la section locale de la SAANB en ayant fait une priorité et ayant très récemment réussi à obtenir la mise sur pied d'un comité chargé de la question du paysage linguistique $^{18}$. D'aucuns préconisent qu'on légifère en la matière, d'autres favorisent des programmes d'incitation auprès des commençants et d'autres encore estiment qu'aucun effort ne devrait être déployé en la matière.

Dans les lignes qui suivent, nous examinerons des extraits d'un entretien fait avec une participante francophone qui a étudié à Moncton et qui y est installée depuis plus de 35 ans, participante qui, dans sa vie professionnelle au sein d'une institution entièrement francophone au moment de sa fondation et où la langue de travail continue à être le français, consacre quand même à l'heure actuelle environ $30 \%$ de son temps à des clients anglophones, selon sa propre estimation ${ }^{19}$. Au sujet de l'environnement linguistique de Moncton, celui-ci s'est considérablement francisé au cours des années dit-elle :

\section{Extrait 1}

Lise est-ce que dans les 30 ans où vous êtes ici est-ce que vous trouvez que la ville a changé

Flora oh oui définitivement

Lise oui

Flora la ville a changé / les premières années quand on est arrivé / surtout quand j'étais étudiante quand j'ai arrivé t'avais pas beaucoup de français partout ou t'allais c'était anglais tu sais nous autres on était étudiantes on restait en 
résidence pis si on allait à un restaurant ou même si on allait à un petit dépanneur il fallait parler anglais y avait personne qui parlait français ça fait que asteure tu peux presque presque là disons pas partout mais assez souvent que tu vas trouver du français même si des fois on a l'erreur de commencer à parler en anglais premier (rires) pis là on s'aperçoit qu'on est en train de parler en anglais avec quelqu'un qui nous parle français / ça fait que / on retourne sur le français // quand même ah oui beaucoup mieux

Lise oui

Flora beaucoup plus de français

Lise donc vous trouvez que c'est une amélioration

Flora ah définitivement définitivement pour tout partout où tu vas au magasin si tu vas à l'épicerie si tu vas à la banque tu trouves toujours quelqu'un pour te parler français

Le lecteur remarquera que la participante a évoqué spontanément la situation linguistique dans sa réponse, alors que la question, qui se situe au début de l'entretien, n'y fait pas allusion. Qui plus est, dans l'explication donnée oralement à la participante en début d'entretien, il n'a pas été question de la situation linguistique; on précise aux participants que la recherche porte sur les représentations de la ville.

Cette même participante, bien qu'elle se réjouisse de la présence accrue du français dans la ville, déclare que ni l'affichage, ni le service en français ne sont des critères qui motivent ses choix de magasins, de restaurants, etc., tout comme la composition linguistique du quartier où elle habite n'a pas été un facteur dans l'achat de sa maison. Il semblerait donc que, pour elle, la recherche d'autres francophones dans le quartier de résidence, de services en français ou de l'affichage en français, en somme, la recherche de marqueurs identitaires dans la ville, importe peu dans l'interaction quotidienne urbaine, la francité étant exprimée dans le milieu 
professionnel et dans les réseaux sociaux, des espaces non liés à la spatialité même de la ville.

Un deuxième participant à l'étude partage cette même attitude. Francis, qui travaille au sein d'un organisme dont la langue de travail est le français, estime qu'il obtient des services satisfaisants en français à Moncton et qu'il n'a plus à revendiquer une place en tant que citoyen de cette langue :

\section{Extrait 2}

Francis et euh moi j'exigeais beaucoup plus peutêtre que j'exige maintenant c'est bizarre peut-être en vieillissant j'exige moins / mais il faut dire aussi que / presque partout on peut se faire servir en français / c'est pas vrai à cent pour cent

[...]

mais je suis plus sûr qu'on a besoin de cette lutte là non plus

[...]

Fait-que maintenant quand je parle en anglais dans les commerces c'est beaucoup plus parce que je sens que bon ben j'ai ma place maintenant fait-que ça me dérange moins

Contrairement à Flora, Francis n'a pas à donner des services en anglais dans son milieu professionnel. De plus, s'il habite un quartier mixte, ses réseaux sociaux et professionnels sont composés entièrement de francophones. Il s'interroge donc sur la nécessité de mener « cette luttelà » encore de nos jours compte tenu de l'amélioration qu'il constate.

C'est un Moncton beaucoup moins «anglais » qu'auparavant qui se dégage de ces deux entretiens menés auprès de francophones, un Moncton où se sont instaurés de nouveaux rapports entre locuteurs des deux communautés linguistiques, le français n'y étant plus la langue honnie d'autrefois. Non seulement il sert de langue de travail pour certains, mais 
aussi il est perçu - et c'est ce point qui est relativement nouveau dans le paysage linguistique de la ville - comme la langue légitime des activités ordinaires de la vie, dans la mesure où l'interaction le permet.

Les réactions des anglophones au paysage linguistique qui les entoure, telles qu'elles se révèlent dans nos entretiens, sont diverses, parfois paradoxales. Sur les 18 anglophones qui ont participé à l'enquête, 14 ont eu des propos sur l'affichage bilingue : 6 se sont déclarés favorables à ce que l'affichage à Moncton soit entièrement bilingue et 8 ont exprimé des réserves, voire de l'opposition, quant aux modalités de l'affichage bilingue. Parmi les réserves exprimées, certains s'opposent à ce que le français soit placé en premier sur les affiches et à ce qu'il y ait des affiches unilingues françaises; d'autres s'opposeraient à ce que l'affichage commercial bilingue devienne une obligation juridique. John, enseignant dans une école secondaire, se dit ouvert au bilinguisme pourvu que les anglophones ne soient pas lésés dans le processus.

\section{Extrait 3}

Cathy $^{20}$ what's your reaction to bilingual signs and labels here?

John (hesitates) uhm/ it frustrates the heck out of me because of/ inconsistencies /for example/ one of the things that really bugs me/ is I know that we try the hardest to have real French and English in all those many places / but/ down at the corner of St. George and / Highfield/ is uh Caisse de populaire/ there is not one word of English / anywhere on the outside of that building / not one / to indicate / you know that they are trying to incorporate you know or whatever /yet // I believe it is expected by the / by French speaking people that on all English establishments they must also have French or whatever /and / like / this is inconsistent.

Cathy humhum 
John you know / you either got to do it all the way or don't do it at all

Sally humhum

Cathy you know and it's just // those kinds of things / irritate me as an English speaking person

On constate que John, inconscient de la place prépondérante qu’occupe la langue anglaise dans l'affichage dans Moncton ${ }^{21}$, exagère celle occupée par l'affichage français. Soit dit en passant que, dans la rue St.-George dont il est question ici, nous avons repéré deux autres coopératives d'épargne et de crédit, entreprises comparables à la Caisse populaire que John cite, dont les devantures sont exclusivement en anglais. La place croissante qu'occupe le français dans le paysage de Moncton, bien qu'il n'occupe pas une place égale à celle qu'occupe l'anglais, entraîne la redéfinition de l'appartenance des espaces, appartenance que l'affichage public rend visible, qui entraîne, à son tour, la redéfinition de la catégorisation sociale, en l'occurrence les catégories espaces-nous / espaces-eux. Dans cette reconfiguration du paysage, la bilinguisation de l'affichage marque l'inclusion croissante des francophones tandis que l'affichage unilingue français, même s’il est beaucoup moins pratiqué, marque explicitement l'appartenance francophone et l'exclusion des anglophones,

De plus, John semble penser que les deux langues sont en réalité présentes de façon équitable partout et qu'il n’existe pas d'inégalités dans l'affichage des deux langues :

\section{Extrait 4}

Cathy do you feel it's important / in Moncton / or in the Moncton area / uhm to have an obvious welcome to French / as well as English // and maybe / uhm / make this obvious through signs or / through services / do you think it's obvious / or do you think we should make it obvious / that we welcome both / languages 


John : I don't know how we could do it any more
/ it's everywhere / it's everywhere //
absolutely everywhere / i/ it it always
amazes me I w/ went to to Maine / over the
Holidays / and and you get / you get shocked
for a while because / you only see English
on all the signs and you keep looking for /
another language on the signs and it's not
there / and you come home / and the minute
you cross the border // you know it's / you
know welcome to New Brunswick
Bienvenue à Nouveau-Brunswick /
immediately / you know

Cathy : humhum

John: and that's fine / we're an officially bilingual province you accept that you know whatever / uhm / I don't know how we could be any more welcoming / we're obvious / that / here we speak English and French /

Ces commentaires, typiques du discours de l'exagération et repérés sous différentes formes et à des degrés divers dans une proportion significative des entretiens, révèlent les représentations cultivées par les anglophones à l'égard de la place, plus imaginaire que réelle, qu’occupent le français et les francophones dans l'espace public monctonien. Ces représentations façonnent les opinions les plus répandues sur la situation linguistique de la ville. Mais comment interpréter ces représentations et comment expliquer leur émergence?

Pour répondre à cette question, il faut évoquer les autres discours qui circulent sur les langues dans la ville (ainsi que dans l'ensemble de la province, sauf qu'ils sont plus prégnants à Moncton où s'actualise l'identité urbaine acadienne et où se superposent les trois régimes linguistiques dont il a été question précédemment). En effet, la presse anglophone et le gouvernement provincial sont particulièrement silencieux sur le fait que les francophones n’aient pas encore réalisé leur pleine égalité, malgré la mise en place du bilinguisme officiel et des changements sociaux qui se sont produits au sein de la communauté 
acadienne. Le discours officiel sur le bilinguisme véhiculé par les médias et le gouvernement, discours qui tend à devenir dominant, chante les louanges de la « fusion » des deux communautés linguistiques, le mélange harmonieux de deux cultures qui ouvre la voie à un nouveau type de société, à de nouvelles façons de faire. Rappelons que l'essor économique que connaît Moncton depuis un certain nombre d'années est directement attribuable au bilinguisme d'une partie de sa population et, donc, à sa capacité d'attirer des industries du secteur tertiaire.

Or, dans l'extrait 5 ci-dessus, si John reprend à son compte le discours véhiculé par la presse sur le bilinguisme idéal dont la ville de Moncton serait l'exemple parfait, les inégalités sociales qui persistent sont passées sous silence. Les conflits du passé autour de la question du bilinguisme, parmi les plus intenses au Canada, sont également gommés. La politique du quotidien anglophone de Moncton de ne plus faire allusion à l'identité linguistique des gens, adoptée en 1997 et citée ci-dessous, est une stratégie discursive qui s’inscrit dans ce discours de l'harmonie.

Our editors decided there would be no more "phone" calls. We don't mean the "allo, how are you?" kind of telephone call.

We refer to the use of "phone" as a label, as in "anglophone" and "francophone".

In Canada, where the description "New Brunswicker" evokes the image of a person capable of living harmoniously with dual cultures and languages our peculiar habit of using these adjectives seems counter-productive to what we represent.

It is time we stopped segregating ourselves with adjectives.

Given that it is inappropriate to describe an individual as "a black New Brunswicker" or "a Chinese New Brunswicker," why should it be acceptable to use anglophone and francophone to stereotype people? (Edith Robb, Times and Transcript, 25 octobre 1997). 
Cette stratégie, dont les intentions sont compréhensibles et qui peuvent paraître louables pour qui croit à la négation des différences, a pour effet de neutraliser les débats entourant la question linguistique au NouveauBrunswick, et plus particulièrement à Moncton. Le discours dominant véhiculé dans la presse anglophone et par les autorités selon lequel les Néo-Brunswickois (et les Monctoniens) vivent sans tensions, construit ainsi la perception que se font les anglophones et les francophones de la réalité linguistique. Si le discours construit la réalité, ou une partie de la réalité, l'institutionnalisation du discours de l'harmonie dément l'aspect conflictuel de la question linguistique et, surtout, laisse voir que tous les locuteurs sont en effet égaux dans leurs droits. Par conséquent, quand la question linguistique est abordée, les anglophones ont tendance à se percevoir comme les laissés-pour-compte :

\section{Extrait 5}

Myrna it doesn't matter how bilingual you are, it's being French that counts

Et, du côté des francophones, on s’attarde plutôt sur les progrès marqués depuis une génération, les réussites du bilinguisme et leur nouveau prestige au sein de la ville. Il se dégage donc de ce partage du territoire urbain par deux communautés linguistiques une double mise en mots de la ville selon qu'on est francophone ou anglophone (voir aussi les conclusions de Lord, 2004, en ce qui a trait à la production littéraire issue des deux communautés linguistiques), l'une s'articulant autour de l'inclusion, l'autre de l'exclusion.

\section{Les écrivains et Moncton}

Pour ajouter à cette recherche, nous avons interrogé les textes de quelques écrivains francophones ${ }^{22}$ qui ont écrit sur Moncton. Gérald Leblanc est reconnu comme le poète qui a chanté Moncton autant dans les chansons qu'il a écrites pour $1755^{23}$ que dans ses textes poétiques ou son roman $^{24}$. Moncton y est dépeint comme le lieu où s'expriment les interrogations identitaires, les contradictions et les ambivalences linguistiques (le bonheur de jouer de plusieurs langues, la frustration liée au bilinguisme asymétrique, le plaisir du chiac, la révolte contre l’unilinguisme pratiqué dans la région). Leblanc traite de la marginalité 
des francophones, marginalité assumée et vécue comme force créatrice qui permet de faire de Moncton un espace habitable : « de Moncton / ville d'automne cet automne / ville de nuit au noir soyeux / où l'on rêve ensemble / d'un espace habitable / où circuler exactement » (Leblanc, 1995 : 89). Sa pensée s’apparente à celle de François Paré qui, dans son essai intitulé La distance habitée, affirme que «les cultures minoritaires sont poussées à négocier de nouveaux espaces composites, parfois juxtaposés, parfois créoles, au sein des ensembles hégémoniques et uniformisants dans lesquels elles se situent inévitablement » (Paré, 2003 : 11). Paré compare les minoritaires aux étrangers, à ces gens venus d'ailleurs qui « ont appris à vivre dans la privation de l'origine, à habiter la distance, comme si leurs mots et leurs langages mêmes avaient depuis toujours inspiré cette fuite en avant" (Ibid.: 9; c'est nous qui soulignons). Dans Moncton Mantra, roman sur Moncton où s'éclatent des personnages qui cherchent à créer des espaces où vivre leur francité, Leblanc montre que cette distance à habiter, si étendue et cahoteuse soitelle, reste le chemin privilégié de son inspiration : «Habiter une ville, avoir un rapport équivoque avec cette ville, teinté d'une sorte d'amourhaine la plupart du temps, mais qui fournit un lieu d'exploration tout de même. Moncton, c'est ça pour moi » (Leblanc, 1997: 112). Ce lieu d'exploration, de pulsions antagonistes drapées de tension créatrice, oblige à une vigilance et à une interrogation constante exprimées dans le poème multipiste.

\section{multipiste}

Moncton serait cet espace

où j'ai traversé le rouge dans toutes ses dimensions

réseaux dévorants des accidents de parcours

rages et rumeurs de l'émotion

ville de mes vies parallèles

Moncton multipiste

dans l'immense Amérique de mon désir

(Leblanc, 1988 : 106) 
Le pouvoir de création qui se donne à voir dans les villes marquées par l’hétérogénéité des cultures et des langues ne masque pas les immenses frustrations engendrées par l'unilinguisme ambiant (dégagé des affiches, supra), frustrations qui se transposent dans les activités ordinaires de la vie.

Anne-Marie arrive de bonne heure sur le coup de midi. Nous descendons au Highfield Square chercher des disques à bon marché. On s’arrête aux Terrasses, le temps d'un café. Au comptoir, Anne-Marie demande deux cafés.

-I don’t speak French! crache un épouvantail déguisé en serveuse.

-Of course you don't, que je lui réponds, whoever heard of peasants speaking two languages.

Anne-Marie et moi gesticulons avec l'index et le majeur, en articulant exagérément : «DEUX CAFÉS! » (Leblanc, 1997 : 136).

La schizophrénie linguistique est l'habitat de nombreux locuteurs francophones monctoniens qui ne savent jamais dans quelle langue s'adresser en public, ni dans quelle langue on leur répondra. Mais cet état, vécu de façon douloureuse par certains, est vécu par d'autres sur le mode de l'humour, sans doute dans une tentative de transgresser l'unilinguisme ambiant, comme dans le poème de Paul Bossé intitulé Pas no:

STOP

ARRÊT

je freine

FISHBONE

ARETE

je m'étouffe

IT’S NICE TO LIVE IN A BILINGUAL CITY

C'est veau de bivre dans une ville si dingue

j'explose

(Bossé, 2001 : 27) 
Dyane Léger n'exprime pas autre chose lorsqu'elle évoque sa vie au quotidien à Moncton. Le fait de vivre et d'écrire en français dans une ville qui laisse peu de place à son existence en tant que francophone est pour elle source de frustrations mais, en même temps, source de stimulation et d'inspiration.

La vie m’enthousiasme plus que jamais. Je vis. J'écris. Toujours en français dans la ville qui porte le nom de celui qui croit m'avoir anéanti. Mon choix reste une revendication. Je le sens dès que je m'aventure hors des murets du ghetto. Les mêmes vieux fantômes qui ont hanté mon enfance réapparaissent. L'acadien, surpris d'entendre mon enfant parler «le bon français » me répète : " ah ben toi c'est pas pareil, t'as eu la chance de sortir, d'aller à l'université »; et l'autre, l'anglophone, scandalisé parce qu'à 5 ans mon enfant ne parle pas comme lui, me questionne: " where do you come from? You are not from here.(..) ». Pas facile l'héritage que je laisse à mon enfant. (...) Jusque là, ça va, il y a encore une voix qui répond : «Oui, je peux vous aider? Après m'avoir dit : I'll get you someone that speaks french » (Léger, 2004 : 248).

Léger montre ici la difficulté de s’actualiser en tant que francophone à Moncton dans ses espaces marqués par la rupture, l'instabilité, le mouvant, l'insécurité linguistique, tout en exprimant un désir d’investir ce lieu où le tumulte de l'incertitude se transpose en souffle créateur (« la vie m’enthousiasme plus que jamais »). Elle montre également la dimension triglossique de Moncton où les Acadiens ont toujours à conjuguer avec le regard de l'autre sur leur langue, que ce soit l'autre - le même, le francophone qui crée une frontière entre lui et celui qui parle une autre forme de français, ou encore que ce soit l'autre, l'anglophone, dont l'imaginaire unilingue admet difficilement une autre langue. Si la dernière voix la rassure " oui je peux vous aider », l'auteure demeure inquiète quant à l'avenir du français dans la ville : « jusque là, ça va ».

Ces courts extraits montrent que les discours qui circulent dans la ville « font » la ville non seulement métaphoriquement mais performativement 
(Mondada, 2000 : 38). La mise en mots de Moncton par les écrivains, celle de ses fractures, de ses incohérences, de ses frontières (imaginaires ou réelles), permet de reterritorialiser la ville dans les représentations, mais cette reterritorialisation n'est pas seulement un produit de l'imaginaire, elle construit véritablement les lieux selon un découpage linguistique façonné à même les mots des poètes (et autres). Ainsi, tel bar ou tel restaurant deviendra francophone ou anglophone, ou les deux, sans lien avec le nom affiché sur la devanture, porté par les discours qui le fabriquent. Dans une ville comme Moncton, davantage marquée par les réseaux linguistiques que par des quartiers bien délimités, si les lieux deviennent habitables pour les locuteurs en manque d'espaces où dire leur être parlant, c'est grâce aux discours qui créent ces lieux comme des espaces pour soi, ce pour soi n'étant pas ici interprété comme un entre soi composé uniquement de mêmes mais aussi des autres qui en acceptent l’hétérogénéité.

\section{Conclusion}

Très concrètement, au moyen d'une analyse exhaustive de l'affichage dans deux rues de Moncton, nous avons pu constater que la langue anglaise domine dans l'affichage et nous pensons pouvoir étendre les conclusions de ce bref recensement à l'ensemble de la ville. Nous avons montré également que les représentations des citoyens de Moncton, surtout celles des anglophones, sont marquées par l'ambivalence et l'ambiguïté et souvent éloignées de la réalité ; en effet, l'exagération de la présence francophone dans la ville, très présente dans les propos des anglophones, semble une constante. Par ailleurs, l'imaginaire des francophones est lui aussi meublé de fantasmes contradictoires comme l'ont montré en particulier les textes des écrivains. Les écrivains cependant se servent de ces contradictions, de cette incertitude pour construire, par leurs mots, un lieu imaginaire et/ou réel où s'exprimer.

Au terme de cette trop brève étude, il nous paraît évident que l'étude des représentations linguistiques revêt un intérêt indéniable pour les études sociolinguistiques qui tendent à définir la linguistique "non à partir des seuls 'faits linguistiques', mais à partir de comportements et de faits, les représentations faisant partie intégrante de ces comportements » (Robillard, 2001: 171). En effet, les représentations linguistiques, 
tributaires des conditions sociales, politiques et économiques dans lesquelles vivent les communautés, participent des positionnements qu'adoptent les locuteurs par rapport à eux-mêmes et par rapport aux autres; en d'autres termes, elles contribuent largement à la construction du soi et de l'autre et jouent un rôle très important dans le changement linguistique. Nous espérons en avoir montré l'importance dans cet article.

\section{Bibliographie}

Allain, G. (à paraître). La nouvelle capitale acadienne ? Les entrepreneurs acadiens et la croissance récente du Grand Moncton. Francophonies d'Amérique. 18.

Barbéris, J.-M. (1999). Analyser les discours. Le cas de l'interview sociolinguistique. In Calvet, L.-J. et Dumont, P. (dir.). L'enquête sociolinguistique. Paris : L’Harmattan. 125-148.

Baudrillard, J. (1986). Amérique. Paris : Grasset.

Beaudin, M. et Higgins, B. (1988). Impact de l'Université de Moncton sur les régions de Moncton, d'Edmundston et de Shippagan. Moncton: Institut canadien de recherche sur le développement régional.

Bossé, P. (2001). Un cendrier plein d'ancêtres. Moncton : Les Éditions Perce-Neige.

Boudreau, A. (2003). Construction identitaire et espace urbain : le cas des Acadiens de Moncton. In Bulot, $\mathrm{T}$. et Messaoudi, $\mathrm{L}$ (dir.). Sociolinguistique urbaine: frontières et territoires. Cortil-Wodon: Éditions Modulaires Européennes. 171-204.

Boudreau, A. (2005). Le français en Acadie : maintien et revitalisation du français dans les provinces Maritimes. In Valdman, A., Auger, J. \& Piston-Hatlen, D. (éd.). Le français en Amérique du Nord; état présent. Ste-Foy : Presses de l'Université Laval. 439-454.

Boudreau, A. et Dubois, L. (2001). Langues minoritaires et espaces publics : le cas de l'Acadie. Estudios de sociolingüística. 2:1.37-60.

Boudreau, A. et Dubois, L. (2003). Les anglophones et le français à Moncton. In Stebbins, R., Romney, C., Ouellet M. (dir.). Francophonie et langue dans un monde divers en évolution : contacts 
interlinguistiques et socioculturels. Winnipeg: Presses universitaires de Saint-Boniface. 143-158.

Bourhis, R. et Landry, R. (2002). La loi 101 et l'aménagement du paysage linguistique au Québec. Revue d'aménagement linguistique. Québec : Office québécois de la langue française. Numéro hors série : 107-132.

Bulot, T. (2002). La double articulation de la spatialité urbaine : « espaces urbanisés » et «lieux de ville» en sociolinguistique. Marges linguistiques. 3:91-105. [http://www.marges-linguistiques.com].

Bulot, T. et Veschambre, V. (2004). Sociolinguistique urbaine et géographie sociale: hétérogénéité des langues et des espaces. Communication au colloque Espaces et société aujourd'hui : la géographie sociale dans les sciences sociales et dans l'action. Rennes. [http://eso.cnrs.fr/evenements/resumes_10_2004.html].

Calvet, L.-J. (1999). Pour une écologie des langues du monde. Plon : Paris.

Dubois, L. (2003). Le bilinguisme à Moncton : lieu de divergence (2003). In Bulot, T. et Messaoudi, L (dir.). Sociolinguistique urbaine: frontières et territoires. Cortil-Wodon: Éditions Modulaires Européennes. 137-170.

Durand, M. (2004). Évolution et consolidation de l'espace francophone du Grand Moncton au Nouveau-Brunswick: 1960-2002. Thèse de maîtrise soutenue en sciences géographiques, Université Laval [http://www.theses.ulaval.ca/2004/21595/21595.html].

Gilbert, A. (1999). Espaces franco-ontariens. Essai. Ottawa : Les Éditions du Nordir.

Heller, M. et Labrie, N. (dir.) (2003). Discours et identités. La francité canadienne entre modernité et mondialisation. Cortil-Wodon : Éditions Modulaires Européennes [coll. Proximités - Sciences du langage]. 12-39 ; 403-419.

Landry, R. et Bourhis, R. (1997). Linguistic landscape and ethnolinguistic vitality. An Empirical Study. Journal of Language and Social Psychology. 16:1.23-49.

Leblanc, G. (1995) Éloge du chiac. Moncton : Les Éditions Perce-Neige. 
Leblanc, G. (1997). Moncton Mantra. Moncton: Les Éditions PerceNeige.

LeBlanc, M. (2003). Les odonymes de la ville de Moncton ... ou la traduction à coups de dictionnaire. In Boudreau, A., Dubois, L., Maurais, J., McConnell, G. (dir.). Actes du Colloque international sur l'écologie des langues. Paris : L'Harmattan. 245-260.

Léger, D. (2004) Intertexte. Le présent... In Klaus, P. (dir.). Neue Romania, Acadie 1604-2004. 240-249.

Lord, M.-L. (2004). La réalité mitoyenne du Moncton postmoderne : bilinguisme et diversité culturelle. Nos diverses cités. 1.93-96.

Mondada, L. (2000). Décrire la ville. La construction des savoirs urbains dans l'interaction et dans le texte. Paris : Anthropos (Éd. Economica) [coll. Villes].

Paré, F. (2003). La distance habitée. Ottawa : Les Édition du Nordir.

Robillard, D. de (2001). Peut-on construire des 'faits linguistiques' comme chaotiques? Quelques éléments de réflexion pour amorcer le débat. Marges linguistiques. 1:163-203. [http://www.margeslinguistiques.com].

Vincent, G. (1992). Une nouvelle centralité urbaine au Canada ? Thèse de doctorat en géographie. Université Laval.

La Loi sur les langues officielles du Canada (1969) et (1989); la Loi sur les langues officielles du Nouveau-Brunswick (1969) et (2002).

2 Organisme d'envergure provinciale voué à la défense et à l'épanouissement des droits des Acadiennes et Acadiens du Nouveau-Brunswick.

3 Le Conseil de l'aménagement linguistique du Nouveau-Brunswick, organisme voué à la promotion et à la facilitation de l'aménagement linguistique dans la province, et dont les deux auteures sont membres fondatrices, s'est donné comme un de ses objectifs d'inciter l'adoption de politiques favorisant l'affichage public dans les deux langues officielles, y compris les domaines de la toponymie, de l'odonymie et du paysage commercial.

4 Moncton s'est donné le statut de ville officiellement bilingue en 2002 ; au moment d'écrire ces lignes, elle était toujours la seule ville canadienne à s'être donné ce statut, bien qu’Ottawa, la capitale nationale, se soit engagée dans le processus. De plus, nous avons limité notre démarche à Moncton, c'est-à-dire que l'affichage inventorié se situe à Moncton et les participants à l'enquête habitent tous Moncton. Toutefois, nous sommes très conscientes que les frontières entre les trois villes qui constituent le Grand Moncton (Moncton, Dieppe et Riverview) sont loin d'être étanches sur tous les plans. 
5 Voir LeBlanc (2003) pour une étude sur l'odonymie et infra pour un relevé de l'affichage public le long de deux axes commerciaux de Moncton.

6 Concepts empruntés à Barbéris, J.-M. (1999).

7 Le projet Prise de parole a été financé de 1996 à 2000 par le Conseil de recherche en sciences humaines du Canada (chercheurs principaux : Normand Labrie, Monica Heller, Université de Toronto et Jürgen Erfurt, Johan Wolfgang Goethe-Universität, Frankfurt a Main ; collaboratrices : Annette Boudreau et Lise Dubois, Université de Moncton), le programme Transcoop de la German-American Academic Council Foundation (chercheurs principaux : Jürgen Erfurt, Monica Heller et Normand Labrie) et l'Agence universitaire de la Francophonie (chercheurs principaux : Patrice Brasseur et Claudine Moïse, Université d’Avignon et des Pays de Vaucluse).

8 Les 8 premiers entretiens ont été réalisés au cours de l'année 2001-2002 ; 8 autres participants ont été interviewés au cours de 2002-2003 ; et enfin 4 ont été interviewés et accompagnés au cours de 2004, dont deux anglophones et deux francophones. Chercheures principales : Annette Boudreau et Lise Dubois; assistantes de recherche: Cathy Rogers, Amy Jo Coleman et Mireille McLaughlin.

9 Le projet Prise de parole a été suivi par un deuxième projet intitulé Prise de parole 2. Cette deuxième partie a été axée sur la nouvelle économie et la francophonie canadienne ; à Moncton, la recherche s'est déroulée dans un centre d'appels où nous avons fait 22 entretiens et plus de 70 heures d'observation.

10 Le budget annuel de l'Université se situe à l'heure actuelle autour de 80 millions \$ et son effectif tourne autour de mille employés. Elle amène dans la ville une masse étudiante de près de 4000 . Selon une étude menée sur l'incidence économique de l'Université de Moncton pour l'année 1986-1987, on évalue à plus de 56 millions \$ les retombées économiques sur le Grand Moncton (Beaudin et Higgins, 1988).

11 Les formations artistiques qu'offre l'Université de Moncton sont sans aucun doute à l'origine de l'effervescence du secteur culturel et artistique de Moncton, et dans une large mesure, des autres régions francophones de la province, les diplômés de ces formations (musique, arts visuels et art dramatique) ayant contribué à la création d’une coopérative artistique, d’une industrie de la musique francophone ainsi que de troupes de théâtre tant traditionnelles qu'alternatives. Fait intéressant à noter : on ne retrouve pas le même dynamisme pour les arts dans la communauté anglophone du Grand Moncton. Voir Boudreau, 2003 et 2005.

12 Dans les faits, les marchés linguistiques de Moncton sont beaucoup plus complexes que ce que nous présentons ici. En effet, le vernaculaire français occupe une place importante dans les échanges linguistiques. Mais comme le sujet principal ici est l'affichage, nous nous limitons à la présence « visible » des deux langues officielles. Pour une description plus étoffée de ces marchés linguistiques, voir Boudreau et Dubois, 2001.

13 Le territoire couvert par ce relevé commence à l'angle de la rue Morton et de l'avenue de l'Université et va jusqu'à l'angle des rues Archibald (l'avenue de l'Université se transformant en rue Archibald) et St.-George ; le long de la rue St.-George, le territoire couvert commence au boulevard Vaughn Harvey et va jusqu'à la rue King. Il est important de souligner que la portion de la rue Archibald qui longe le campus de l’Université de Moncton porte le nom de l'avenue de l’Université depuis 1999 seulement.

14 L'exception constitue un organisme relevant de l'ambassade de France au Canada.

15 Le territoire couvert par ce relevé le long de cette rue commence à la rue King et va jusqu'au passage du chemin de fer, communément appelé à Moncton le subway.

16 Dans les deux cas, les entreprises « locales » affichant en anglais sont plus nombreuses : environ $90 \%$ dans St.-George/Archibald-avenue de l’Université et 80 \% dans la rue Main.

17 Nous faisons remarquer ici que le mot café est presque toujours orthographié avec le é. 
18 À noter que ce n’est pas la première fois dans l'histoire de Moncton qu'un comité ait été mis sur pied pour étudier la question du paysage linguistique. En 1999, en prévision du Sommet international de la francophonie, un comité avait été formé pour étudier la question de l'odonymie.

19 Dans une perspective aménagiste, on fait la distinction entre langue de travail, qui sert à la communication entre la direction et les employés, ainsi qu'entre les employés, et la langue de service, qui sert à la communication entre le fournisseur du service et le consommateur de ce service. Dans ce cas-ci, le français est langue de travail ; la langue de service est celle demandée par le consommateur et est ou bien le français, ou bien l'anglais.

20 Prénom de l'assistante de recherche qui a fait les entrevues.

21 On peut avancer que l'extrait reflète l'opinion d'un nombre important d'anglophones de la ville, opinion repérable dans des lettres à l'opinion du lecteur publiées dans le quotidien anglophone de Moncton.

22 Nous n'avons rien trouvé du côté anglophone sur Moncton. Cette absence nous paraît significative et conforme aux commentaires des anglophones et des francophones qui ont fait état de la vitalité de la communauté artistique francophone à Moncton depuis les vingt dernières années. Nous remercions Glen Nichols du Département d'anglais de l’Université de Moncton d'avoir discuté de la question avec nous.

23 Groupe de musique populaire des années soixante-dix qui a connu et qui connaît encore un succès très important.

24 Il a publié une dizaine de recueils de poésie et un roman intitulé Moncton Mantra en 1997. 